PROCEEDINGS OF THE

AMERICAN MATHEMATICAL SOCIETY

Volume 135, Number 8, August 2007, Pages 2403-2414

S 0002-9939(07)08768-0

Article electronically published on April 10, 2007

\title{
SIMPLICIAL SHELLABLE SPHERES VIA COMBINATORIAL BLOWUPS
}

\author{
SONJA LJ. ČUKIĆ AND EMANUELE DELUCCHI
}

(Communicated by Paul Goerss)

\begin{abstract}
The construction of the Bier sphere $\operatorname{Bier}(K)$ for a simplicial complex $K$ is due to Bier (1992). Björner, Paffenholz, Sjöstrand and Ziegler (2005) generalize this construction to obtain a $\operatorname{Bier}$ poset $\operatorname{Bier}(P, I)$ from any bounded poset $P$ and any proper ideal $I \subseteq P$. They show shellability of $\operatorname{Bier}(P, I)$ for the case $P=B_{n}$, the boolean lattice, and thereby obtain 'many shellable spheres' in the sense of Kalai (1988).

We put the Bier construction into the general framework of the theory of nested set complexes of Feichtner and Kozlov (2004). We obtain 'more shellable spheres' by proving the general statement that combinatorial blowups, hence stellar subdivisions, preserve shellability.
\end{abstract}

\section{INTRODUCTION}

Let $K$ denote an abstract simplicial complex on the vertex set $[n]:=\{1, \ldots, n\}$, and write $\mathcal{F}(K)$ for the poset of its faces. The (combinatorial) Alexander dual for $K$ is the simplicial complex $A(K)$ whose faces are the complements (in $[n]$ ) of the nonfaces of $K$. Thus, $A(K)=\left\{[n] \backslash \sigma \mid \sigma \in 2^{[n]} \backslash K\right\}$. The topological motivation for this suggestive name is that, in fact, $K$ and $A(K)$ can be 'put together' to a sphere. A very nice construction of this sphere is due to Thomas Bier $[\mathrm{B}$ and can be found in [M, p. 111-116]. Bier showed that the deleted join $\operatorname{Bier}_{n}(K):=(K * A(K))_{\Delta}$ is an $(n-2)$-sphere with at most $2 n$ vertices $[\mathrm{M}$, Theorem 5.6.2]. The idea behind this proof is to embed $\mathcal{F}(K)$ in the boolean lattice $B_{n}$ and see that $\operatorname{Bier}_{n}(K)$ is in fact a subdivision of the boundary of $\Delta\left(B_{n}\right)$.

Björner et al. generalize this construction in BPSZ to obtain a Bier poset $\operatorname{Bier}(I, P)$ associated to any proper lower ideal $I$ in any bounded poset $P$. They show that, for any such $P$, the order complex of $\overline{\operatorname{Bier}(P, I)}$ is PL-homeomorphic to that of $\bar{P}$. In the boolean case $P=B_{n}$ we have $\operatorname{Bier}\left(B_{n}, I\right)=\operatorname{Bier}_{n}(I)$ (at the right hand side of the equality $I$ is seen as an abstract simplicial complex). In the same paper shellability of $\operatorname{Bier}\left(B_{n}, I\right)$ is proven, together with a characterization of its $g$-vector. In particular, it is pointed out that, for large $n$, this construction

Received by the editors February 2, 2006 and, in revised form, May 2, 2006.

2000 Mathematics Subject Classification. Primary 06A07, 55U10, 52B22.

Key words and phrases. Posets, lattices, shellability, combinatorial blowups, building sets, nested sets, simplicial shellable spheres, Bier posets, Bier lattices.

Research partially supported by TH-Projekt 0-20268-05, and by the Swiss National Science Foundation, project PP002-106403/1.

(C)2007 American Mathematical Society Reverts to public domain 28 years from publication 
leads to 'many simplicial shellable $(n-2)$-spheres', most of them lacking convex realization (see $\mathrm{Ka}]$ ).

We put this construction in the context of the theory of nested set complexes (developed by Feichtner and Kozlov in FK1]). Specifically, we find a conceptual way of proving that $P$ and $\operatorname{Bier}(P, I)$ are PL-homeomorphic if $P$ is a lattice. Moreover, we obtain shellability of $\operatorname{Bier}(P, I)$ for any shellable lattice $P$ and 'even more' simplicial shellable spheres.

In section 1 we introduce notation and define the basic characters of this paper. Section 2 relates the poset $P$ to a building set in the poset $\operatorname{Bier}(P, I)$ and then, restricting to the case when $\operatorname{Bier}(P, I)$ is a semilattice, shows how combinatorial blowups relate order complexes of $\bar{P}$ and $\overline{\operatorname{Bier}(P, I)}$. The core of section 3 is Theorem 3.4 where we show that combinatorial blowups preserve shellability. This applies in particular to the Bier construction and implies that $\operatorname{Bier}(P, I)$ is shellable and homotopy equivalent to a wedge of spheres whenever $P$ is a shellable poset. Note that this even allows us to iterate the Bier construction to get shellable triangulations of spheres with any number of vertices, whose isomorphism type depends on $P, I$, and the number of iterations.

\section{Notation, Definitions AND BAsic PROperties}

1.1. Posets. In this section we will give a summary of the standard definitions and notation which will be used further in the paper. For a general reference to the theory of posets and lattices, we refer the reader to [Sta, Chapter 3].

Let $(P, \leq)$ be a poset. All posets considered in this paper will be finite. We call $P$ bounded if there exist elements $\hat{0}, \hat{1} \in P$ so that $\hat{0} \leq x \leq \hat{1}$ for all $x \in P$. We will write $\bar{P}$ for the proper part of $P$, that is $\bar{P}=P \backslash\{\hat{0}, \hat{1}\}$. Also, let $P_{\leq x}=$ $\{y \in P \mid y \leq x\}$. Similarly, for any $\mathcal{G} \subseteq P$ with order induced by $P$, let us write $\mathcal{G}_{\leq x}=\{y \in P \mid y \in \mathcal{G}, y \leq x\}$. We say that $y$ covers $x$ if $y>x$ and there is no $z \in P$ so that $y>z>x$; in this case we will write $x \lessdot y$. For $x \leq y$, the interval $\{z \in P \mid x \leq z \leq y\}$ is denoted by $[x, y]$. A subset $I \subseteq P$ is an ideal in $P$ if, for all $y \in I$ and $x \in P, x \leq y$, then $x \in I$. An ideal $I$ is called proper if $I \neq P$ and $I \neq \emptyset$. With $\Delta(P)$ we will denote the order complex of the given poset $P$ : the abstract simplicial complex whose vertices are the elements of $P$, and faces are all chains in $P$ (including the empty chain). In this paper we will assume that the empty face is an element of every non-empty abstract simplicial complex.

A poset $\mathcal{L}$ is called a meet-semilattice, or simply semilattice, if every pair of elements $x, y \in \mathcal{L}$ has a unique maximal lower bound, which is called a meet of these two elements, and is denoted by $x \wedge y$. All semilattices have a unique minimal element called $\hat{0}$, and for any $A=\left\{a_{1}, \ldots, a_{t}\right\} \subseteq \mathcal{L}$, the set $\{x \in \mathcal{L} \mid x \geq$ $a_{i}$, for all $\left.i \in[t]\right\}$ is either empty, or it has a unique minimal element, its join, $\bigvee A=a_{1} \vee \cdots \vee a_{t}$. Finally, a semilattice $L$ is a lattice if meet and join are defined for any pair of elements of $L$.

Definition 1.1 ([BPSZ, Definition 1.1]). Let $P$ be a bounded poset of finite length and $I \subset P$ a proper ideal. Then the poset $\operatorname{Bier}(P, I)$ is defined as follows:

- elements are all intervals $[x, y] \subseteq P$ such that $x \in I$ and $y \notin I$, together with an additional top element $\hat{1}$,

- intervals are ordered by reverse inclusion, i.e., $[x, y] \leq[v, w]$ if and only if $x \leq v<w \leq y$. 


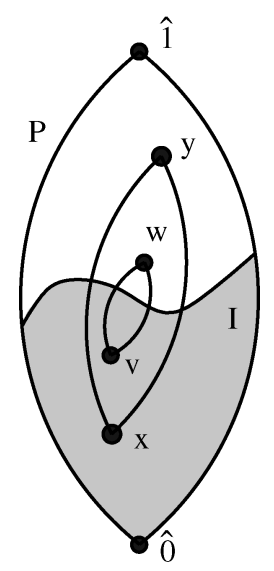

Figure 1. Two elements $[x, y]$ and $[v, w]$ of $\operatorname{Bier}(P, I)$ with $[x, y] \leq[v, w]$.

Clearly, $\operatorname{Bier}(P, I)$ is a bounded poset. Furthermore, by [BPSZ, Lemma 1.2], if $P$ is a lattice, then $\operatorname{Bier}(P, I)$ is also a lattice.

1.2. Building sets, nested sets, combinatorial blowups and stellar subdivisions. The theory of building sets, nested set complexes and combinatorial blowups for general semilattices was initiated and developed by Feichtner and Kozlov, [FK1], as the combinatorial framework of the wonderful models for subspace arrangements by de Concini and Procesi [DCP, F1]. However, this theory has found application in many different contexts, as can be seen in [F2, FK2, FM, FS. We recall here the basic definitions and refer to FK1] for a comprehensive introduction.

Definition 1.2 ([FK1, Definition 2.2]). Let $\mathcal{L}$ be a semilattice. A subset $\mathcal{G}$ of $\mathcal{L} \backslash\{\hat{0}\}$ is called a building set of $\mathcal{L}$ if for any $x \in \mathcal{L} \backslash\{\hat{0}\}$ and $\max \mathcal{G}_{\leq x}=\left\{x_{1}, \ldots, x_{t}\right\}$, there is an isomorphism of posets

$$
\psi_{x}: \prod_{i=1}^{t}\left[\hat{0}, x_{i}\right] \longrightarrow[\hat{0}, x],
$$

such that $\psi_{x}\left(\hat{0}, \ldots, \hat{0}, x_{i}, \hat{0}, \ldots, \hat{0}\right)=x_{i}$, for all $i \in[t]$.

Remark. Note that this definition does not really require the semilattice structure of $\mathcal{L}$. Therefore such an object can be defined in any bounded poset with a unique minimal element. However, if $\mathcal{L}$ is a semilattice, then $\psi_{x}$ can always be chosen to be the canonical map $\left(y_{1}, \ldots, y_{t}\right) \mapsto y_{1} \vee \cdots \vee y_{t}$.

Definition 1.3 ([FK1, Definition 2.7]). Let $\mathcal{L}$ be a semilattice and $\mathcal{G}$ a building set of $\mathcal{L}$. A (possibly empty) subset $N$ of $\mathcal{G}$ is called nested if for any $\left\{x_{1}, \ldots, x_{t}\right\} \subseteq N$, where $t \geq 2$ and any two distinct elements $x_{i}$ and $x_{j}$ are incomparable, the join $x_{1} \vee \cdots \vee x_{t}$ exists and does not belong to $\mathcal{G}$.

The nested sets in $\mathcal{G}$ form an abstract simplicial complex, called the nested set complex of $\mathcal{G}$ in $\mathcal{L}$, and which will be denoted by $\mathcal{N}(\mathcal{L}, \mathcal{G})$.

If it is clear which semilattice $\mathcal{L}$ is meant, we will write $\mathcal{N}(\mathcal{G})$ instead of $\mathcal{N}(\mathcal{L}, \mathcal{G})$. Remark. It is not hard to see that if $\mathcal{G}$ is the maximal building set in the given semilattice $\mathcal{L}$, then $\mathcal{N}(\mathcal{L}, \mathcal{G})=\Delta(\mathcal{L} \backslash\{\hat{0}\})$. 

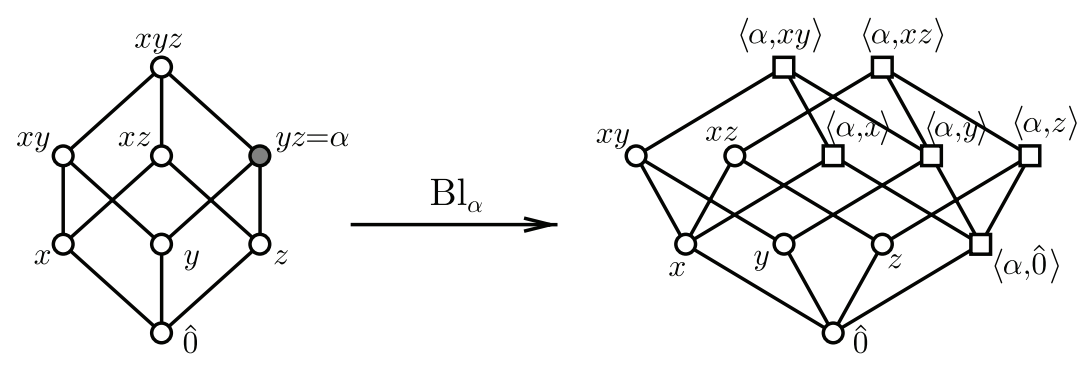

Figure 2. Example of the combinatorial blowup of the boolean lattice $B_{3}$ at the element $\{y, z\}$.

Definition 1.4 ([FK1, Definition 3.1]). For a semilattice $\mathcal{L}$ and an element $\alpha \in \mathcal{L}$ we define a new poset $\mathrm{Bl}_{\alpha} \mathcal{L}$ on the set of elements

$$
\{x \in \mathcal{L} \mid x \nsupseteq \alpha\} \cup\left\{\langle\alpha, x\rangle \mid x \in \mathcal{L}, x \nsupseteq \alpha \text { and }(x \vee \alpha)_{\mathcal{L}} \text { exists }\right\},
$$

with order relation defined as follows:

(i) $y>z$ in $\mathrm{Bl}_{\alpha} \mathcal{L}$ if $y>z$ in $\mathcal{L}$;

(ii) $\langle\alpha, y\rangle>\langle\alpha, z\rangle$ in $\mathrm{Bl}_{\alpha} \mathcal{L}$ if $y>z$ in $\mathcal{L}$;

(iii) $\langle\alpha, y\rangle>z$ in $\mathrm{Bl}_{\alpha} \mathcal{L}$ if $y \geq z$ in $\mathcal{L}$;

where in all three cases $y, z \nsupseteq \alpha$.

The poset $\mathrm{Bl}_{\alpha} \mathcal{L}$ is called the combinatorial blowup of $\mathcal{L}$ at $\alpha$.

Remark. To avoid ambiguities, we will use the notation $\langle\alpha, y\rangle$ instead of $[\alpha, y]$ which was used in [FK1].

Remark. Given a semilattice $\mathcal{L}$ and an element $\alpha \in \mathcal{L}$, the poset $\mathrm{Bl}_{\alpha} \mathcal{L}$ is again a semilattice; see [FK1, Lemma 3.2].

Example. Let $\mathcal{F}=\mathcal{F}(K)$ be the face semilattice of some simplicial complex $K$, that is, elements of $\mathcal{F}(K)$ are faces of $K$, and they are ordered by inclusion (the minimal element of $\mathcal{F}(K)$ is the empty face of $K$ ). Let $\alpha \in \mathcal{F}$ be a face of $K$. By the previous remark, $\mathrm{Bl}_{\alpha} \mathcal{F}$ is again a semilattice. If we have two elements from $\mathrm{Bl}_{\alpha} \mathcal{F}$, let us see what their meet is equal to:

- If $F, G \in \mathcal{F} \cap \mathrm{Bl}_{\alpha} \mathcal{F}$, then $F \wedge G=F \cap G$, seen as an element of $\mathrm{Bl}_{\alpha} \mathcal{F}$.

- If $F,\langle\alpha, G\rangle \in \mathrm{Bl}_{\alpha} \mathcal{F}$, where $F, G \in \mathcal{F}$, then $F \wedge\langle\alpha, G\rangle=F \cap G \in \mathrm{Bl}_{\alpha} \mathcal{F}$.

- Finally, if $\langle\alpha, F\rangle,\langle\alpha, G\rangle \in \mathrm{Bl}_{\alpha} \mathcal{F}, F, G \in \mathcal{F}$, then $\langle\alpha, F\rangle \wedge\langle\alpha, G\rangle=\langle\alpha, F \cap G\rangle$.

We proceed with the definition of stellar subdivision for abstract simplicial complexes. Note that, passing to the geometric realization, this translates to the wellknown corresponding geometrical notion.

Definition 1.5. The stellar subdivision of a simplicial complex $K$ with respect to a non-empty face $F$ is the simplicial complex $\operatorname{sd}_{F}(K)$ whose faces are

$$
\{G \in K \mid G \nsupseteq F\} \cup\left\{G \cup\left\{v_{F}\right\} \mid G \in K, G \nsupseteq F \text {, and } G \cup F \in K\right\} .
$$

Remarks.

- It was noticed in [Ko2, Section 3] that $\mathcal{F}\left(\operatorname{sd}_{F}(K)\right)=\mathrm{Bl}_{F}(\mathcal{F}(K))$, that is, stellar subdivisions are instances of combinatorial blowups.

- It is known that there exists a sequence of elementary collapses and elementary expansions leading from a simplicial complex $K$ to the complex $\operatorname{sd}_{F}(K)$. In other 
words, $K$ and $\operatorname{sd}_{F}(K)$ have the same simple homotopy type; see for example [Ko2, Section 3] for a description of formal deformation from $K$ to $\operatorname{sd}_{F}(K)$.

\section{Building Sets in Bier lattices}

From now on, unless stated otherwise, we will assume that $L$ is a lattice. Then $\operatorname{Bier}(L, I)$ is also a lattice, and we can therefore apply the theory of nested set complexes. We begin by describing a building set in $\operatorname{Bier}(L, I)_{<\hat{1}}$ that is naturally associated to $L$.

Proposition 2.1. For any Bier lattice $\operatorname{Bier}(L, I)$, where $I \subseteq L$ is a proper ideal,

$$
\mathcal{G}:=\{[\hat{0}, y] \mid y \in \bar{L} \backslash I\} \cup\{[x, \hat{1}] \mid x \in I \backslash\{\hat{0}\}\}
$$

is a building set in $\operatorname{Bier}(L, I)_{<\hat{1}}$.

Proof. Let $[x, y] \in \overline{\operatorname{Bier}(L, I)}$. If $[x, y] \in \mathcal{G}$, then Definition 1.2 is clearly satisfied. Suppose then that $[x, y] \notin \mathcal{G}$, i.e., $x \neq \hat{0}$ and $y \neq \hat{1}$. It is clear that

$$
\max \mathcal{G}_{\leq[x, y]}=\{[x, \hat{1}],[\hat{0}, y]\} .
$$

Since in $\operatorname{Bier}(L, I)$ we have that $[[\hat{0}, \hat{1}],[x, \hat{1}]]=\left\{\left[x^{\prime}, \hat{1}\right] \mid x^{\prime} \leq x\right\},[[\hat{0}, \hat{1}],[\hat{0}, y]]=$ $\left\{\left[\hat{0}, y^{\prime}\right] \mid y^{\prime} \geq y\right\}$, and $[[\hat{0}, \hat{1}],[x, y]]=\left\{\left[x^{\prime}, y^{\prime}\right] \mid x^{\prime} \leq x, y^{\prime} \geq y\right\}$, it is easy to see that the function

$$
\begin{aligned}
\psi_{[x, y]}:[[\hat{0}, \hat{1}],[x, \hat{1}]] \times[[\hat{0}, \hat{1}],[\hat{0}, y]] & \longrightarrow[[\hat{0}, \hat{1}],[x, y]], \text { where } \\
\left(\left[x^{\prime}, \hat{1}\right],\left[\hat{0}, y^{\prime}\right]\right) & \longmapsto\left[x^{\prime}, y^{\prime}\right],
\end{aligned}
$$

is an order-preserving bijection of these posets.

It is also easy to see that $\psi_{[x, y]}\left([\hat{0}, \hat{1}],\left[\hat{0}, y^{\prime}\right]\right)=\left[\hat{0}, y^{\prime}\right]$ and $\psi_{[x, y]}\left(\left[x^{\prime}, \hat{1}\right],[\hat{0}, \hat{1}]\right)=$ $\left[x^{\prime}, \hat{1}\right]$. Therefore the function $\psi_{[x, y]}$ satisfies the conditions of Definition 1.2 , and $\mathcal{G}$ is a building set in $\operatorname{Bier}(L, I)_{<\hat{1}}$.

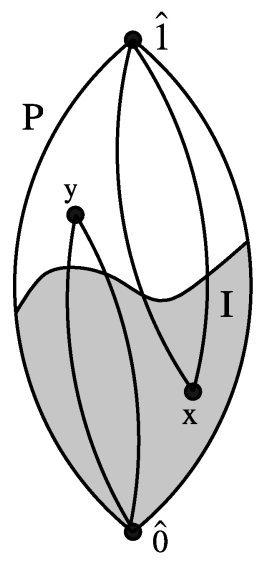

(a)

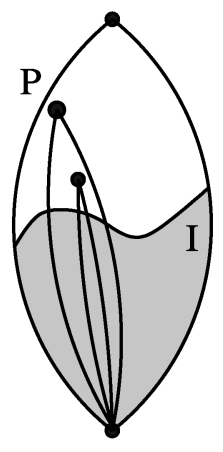

(i)

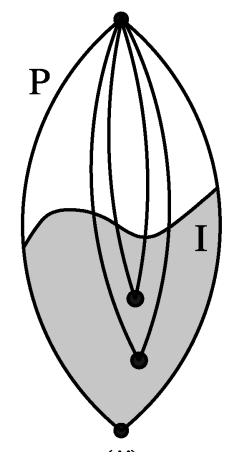

(ii)

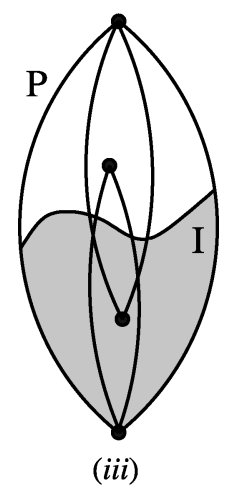

(b)

Figure 3. (a) Elements of the building set $\mathcal{G}$. (b) The three cases considered in Lemma 2.2 . 
It is also true that $\mathcal{G}$ is a building set in $\operatorname{Bier}(L, I)_{<\hat{1}}$ if $L$ is any bounded poset, since the definition we gave is independent of the existence of meet and join operations. However, in that case it is not clear how to characterize the concept of nested sets, even for the special case of Bier posets.

In the lattice case the standard definition works, and therefore we move towards our next goal, the characterization of the nested set complex of $\mathcal{G}$, which will be reached in Proposition 2.3. We need a preparatory lemma.

Lemma 2.2. Let $\mathcal{G}$ be the building set of $\operatorname{Bier}(L, I)_{<\hat{1}}$ defined in Proposition 2.1 . Then a (possibly empty) subset $A$ of $\mathcal{G}$ is nested if and only if the following three conditions are satisfied:

(i) If $\left[\hat{0}, y_{1}\right],\left[\hat{0}, y_{2}\right] \in A$, then $y_{1}$ and $y_{2}$ are comparable in $L$.

(ii) If $\left[x_{1}, \hat{1}\right],\left[x_{2}, \hat{1}\right] \in A$, then $x_{1}$ and $x_{2}$ are comparable in $L$.

(iii) If $[x, \hat{1}],[\hat{0}, y] \in A$, then $x<y$ in $L$.

Proof. ( $\Longrightarrow$ ) Suppose that $A$ is nested, and suppose that (i) is not true, i.e., that there exists a pair of elements $\left[\hat{0}, y_{1}\right],\left[\hat{0}, y_{2}\right] \in A$ such that $y_{1}$ and $y_{2}$ are not comparable. Then $\left\{\left[\hat{0}, y_{1}\right],\left[\hat{0}, y_{2}\right]\right\}$ is a set of incomparable elements in $A$. But

$$
\left[\hat{0}, y_{1}\right] \vee\left[\hat{0}, y_{2}\right]= \begin{cases}{\left[\hat{0}, y_{1} \wedge y_{2}\right] \in \mathcal{G},} & \text { if } y_{1} \wedge y_{2} \notin I \\ \text { doesn't exist, } & \text { otherwise }\end{cases}
$$

This is a contradiction with $A$ being nested. Condition (ii) is proved analogously.

It is left to prove that (iii) is true. Since $[x, \hat{1}]$ and $[\hat{0}, y]$ are incomparable, $[x, \hat{1}] \vee[\hat{0}, y]=[p, q] \in \operatorname{Bier}(L, I)_{<\hat{1}} \backslash \mathcal{G}$, where $x \leq p<q<\hat{1}$ and $\hat{0}<p<q \leq y$. Therefore $x<y$.

$(\Longleftarrow)$ Suppose that $A=\left\{\left[x_{1}, \hat{1}\right], \ldots,\left[x_{l}, \hat{1}\right],\left[\hat{0}, y_{1}\right], \ldots,\left[\hat{0}, y_{k}\right]\right\}$, and $x_{1}<x_{2}<$ $\cdots<x_{l}<y_{1}<\cdots<y_{k}$. If $l=0$ or $k=0$, then $A$ is a chain, and hence nested. Otherwise, it is clear that the cardinality of any set of incomparable elements is at most two. Take any two incomparable elements $\left[x_{p}, \hat{1}\right]$ and $\left[\hat{0}, y_{q}\right]$ from $A$. Then $\left[x_{p}, \hat{1}\right] \vee\left[\hat{0}, y_{q}\right]=\left[x_{p}, y_{q}\right] \notin \mathcal{G}$. Therefore $A$ is a nested set.

The lemma immediately implies the following proposition.

Proposition 2.3. Let $\mathcal{G}$ be the building set of $\operatorname{Bier}(L, I)_{<\hat{1}}$ defined in Proposition 2.1. Then $\mathcal{N}(\mathcal{G})=\Delta(\bar{L})$.

The next proposition is similar in spirit to Proposition 4.2 of [FM], but works in the abstract case as well and does not assume atomicity of the lattice. It describes the behavior of nested set complexes under an extension of the building set.

Proposition 2.4. Let $\mathcal{L}$ be a semilattice, and let $\mathcal{G}$ be a building set in $\mathcal{L}$. If $\alpha \in \max (\mathcal{L} \backslash \mathcal{G})$, then $\mathcal{G}^{\prime}=\mathcal{G} \cup\{\alpha\}$ is a building set in $\mathcal{L}$, and $\mathcal{F}\left(\mathcal{N}\left(\mathcal{G}^{\prime}\right)\right)=$ $\mathrm{Bl}_{B}(\mathcal{F}(\mathcal{N}(\mathcal{G})))$, where $B=\max \mathcal{G}_{\leq \alpha}$.

Proof. Note first that the number of elements of $B$ is at least 2, since otherwise Definition 1.2 would not be satisfied for the building set $\mathcal{G}$ and the element $\alpha \in \mathcal{L}$. If $\left\{\beta_{1}, \ldots, \beta_{t}\right\} \subseteq B$ is an antichain, with $t \geq 2$, then $\beta_{1}<\bigvee_{i=1}^{t} \beta_{i} \leq \alpha$ and hence $\bigvee_{i=1}^{t} \beta_{i} \in \mathcal{L} \backslash \mathcal{G}$. Therefore $B \in \mathcal{N}(\mathcal{G})$. Let us now prove that $\mathcal{G}^{\prime}$ is a building set. Since $\alpha$ is a maximal element of $\mathcal{L} \backslash \mathcal{G}$, it is easy to see that, for all $x \in \mathcal{L} \backslash \mathcal{G}^{\prime}$, $\max \mathcal{G}_{\leq x}^{\prime}=\max \mathcal{G}_{\leq x}$, and for $x \in \mathcal{G}^{\prime}, \max \mathcal{G}_{\leq x}^{\prime}=x$. Since $\mathcal{G}$ is a building set, by Definition $1.2, \mathcal{G}^{\prime}$ is also a building set. 
Now define a map $f: \mathcal{F}\left(\mathcal{N}\left(\mathcal{G}^{\prime}\right)\right) \rightarrow \mathrm{Bl}_{B}(\mathcal{F}(\mathcal{N}(\mathcal{G})))$ in the following way:

$$
f(A)= \begin{cases}A, & \text { if } \alpha \notin A ; \\ \langle B, A \backslash\{\alpha\}\rangle, & \text { otherwise, }\end{cases}
$$

where $A \in \mathcal{F}\left(\mathcal{N}\left(\mathcal{G}^{\prime}\right)\right)$. Let us prove that $f$ is an order-preserving bijection.

- The map $f$ is well-defined:

(1) If $\alpha \notin A$, then let $\left\{a_{1}, \ldots, a_{t}\right\}, t \geq 2$, be a set of incomparable elements in $A$, if such exists. Then $\bigvee_{i=1}^{t} a_{i} \in \mathcal{L} \backslash \mathcal{G}^{\prime} \subset \mathcal{L} \backslash \mathcal{G}$, and hence $A \in \mathcal{N}(\mathcal{G})$. Since $B$ is an antichain with at least two elements, $\bigvee B=\alpha$, and by assumption $A \in \mathcal{N}\left(\mathcal{G}^{\prime}\right)$ it follows that $A \nsupseteq B$. Therefore $f(A)=A \in \mathrm{Bl}_{B}(\mathcal{F}(\mathcal{N}(\mathcal{G}))$ ).

(2) If $\alpha \in A$, then $A \backslash\{\alpha\} \in \mathcal{N}\left(\mathcal{G}^{\prime}\right)$, and therefore $A \backslash\{\alpha\} \in \mathcal{N}(\mathcal{G})$ and $B \nsubseteq$ $A \backslash\{\alpha\}$. In order to prove that $\langle B, A \backslash\{\alpha\}\rangle$ is an element of $\mathrm{Bl}_{B}(\mathcal{F}(\mathcal{N}(\mathcal{G})))$, we need to check that $B \vee(A \backslash\{\alpha\}) \in \mathcal{F}(\mathcal{N}(\mathcal{G}))$, i.e., that $B \cup(A \backslash\{\alpha\})$ is a nested set in $\mathcal{G}$.

Note that, for all $x \in A \backslash\{\alpha\}, x$ has to be comparable with $\alpha$, since otherwise $\{x, \alpha\}$ would be an antichain in $A$, and $x \vee \alpha$ either does not exist, or $x \vee \alpha>\alpha$, and hence $x \vee \alpha \in \mathcal{G}^{\prime}$. This would contradict the fact that $A$ is a nested set in $\mathcal{G}^{\prime}$.

Let $\left\{x_{1}, \ldots, x_{t}\right\}$, where $t \geq 2$, be a set of incomparable elements in $B \cup(A \backslash\{\alpha\})$. If there exists some $i \in[t]$ such that $x_{i} \geq \alpha$, then it is easy to see that $\left\{x_{1}, \ldots, x_{t}\right\} \cap$ $B=\emptyset$, and hence $\left\{x_{1}, \ldots, x_{t}\right\} \subseteq A \backslash\{\alpha\}$. Then $\bigvee_{i=1}^{t} x_{i} \in \mathcal{L} \backslash \mathcal{G}$ follows from the fact that $A \backslash\{\alpha\}$ is nested in $\mathcal{G}$. Suppose now that there exists $i \in[t]$ such that $x_{i} \in B$. In this case we have that $x_{j} \leq \alpha$ for all $j \in[t]$, and $\alpha \geq \bigvee_{j=1}^{t} x_{j}>x_{i}$. Therefore $\bigvee_{j=1}^{t} x_{j} \in \mathcal{L} \backslash \mathcal{G}$. We conclude that $B \cup(A \backslash\{\alpha\}) \in \mathcal{N}(\mathcal{G})$.

- By definition, $f$ is injective.

- The map $f$ is surjective:

If $F \in \mathrm{Bl}_{B}(\mathcal{F}(\mathcal{N}(\mathcal{G})))$ and $F$ is a face of $\mathcal{N}(\mathcal{G})$, then $F \nsupseteq B$. We want to prove that $F \in \mathcal{N}\left(\mathcal{G}^{\prime}\right)$. Let $\left\{y_{1}, \ldots, y_{s}\right\}, s \geq 2$, be a set of incomparable elements in $F$. Since $\bigvee_{i=1}^{s} y_{i} \in \mathcal{L} \backslash \mathcal{G}$, and $\mathcal{G}^{\prime}=\mathcal{G} \cup\{\alpha\}$, we are supposed to prove that $\bigvee_{i=1}^{s} y_{i} \neq \alpha$. Assume the contrary. Then, by [FK1, Proposition 2.8], $\left\{y_{1}, \ldots, y_{s}\right\}=$ $\max \mathcal{G}_{\leq y_{1} \vee \cdots \vee y_{s}}=\max \mathcal{G}_{\leq \alpha}=B$, which is a contradiction with $F \nsupseteq B$. Hence $F \in \mathcal{F}\left(\mathcal{N}\left(\mathcal{G}^{\prime}\right)\right)$, and $f(F)=F$.

If $\langle B, A\rangle \in \mathrm{Bl}_{B}(\mathcal{F}(\mathcal{N}(\mathcal{G})))$, then $A \nsupseteq B$, and $A \cup B \in \mathcal{N}(\mathcal{G})$. It is clear that $\alpha \notin A$, since $\alpha \notin \mathcal{G}$. We ought to prove that $A \cup\{\alpha\}$ is nested in $\mathcal{G}^{\prime}$. Let us first prove that all elements in $A$ are comparable with $\alpha$. Suppose the contrary, i.e., that there exists $y \in A$ so that $y$ is not comparable with $\alpha$. Then $S:=B \backslash \mathcal{L}_{\leq y} \neq \emptyset$, since otherwise $y \geq \bigvee B=\alpha$. Now, if $\alpha \vee y$ exists in $\mathcal{L}$, we would have $\alpha<$ $y \vee \alpha=y \vee \bigvee B=y \vee \bigvee S$, and hence $y \vee \bigvee S \in \mathcal{G}$. This is a contradiction with $A \cup B \in \mathcal{N}(\mathcal{G})$, since $\{y\} \cup S \subseteq A \cup B$ is a set of incomparable elements of cardinality at least two.

Since any set of incomparable elements $\left\{a_{1}, \ldots, a_{s}\right\}$ in $A \cup\{\alpha\}$, where $s \geq 2$, is actually a subset of $A$, by the same arguments as above we conclude that $\bigvee_{j=1}^{s} y_{j} \in$ $\mathcal{L} \backslash \mathcal{G}^{\prime}$. Therefore $A \cup\{\alpha\}$ is nested in $\mathcal{G}^{\prime}$ and $f(A \cup\{\alpha\})=\langle B, A\rangle$.

- It is clear that both $f$ and $f^{-1}$ are order-preserving.

Therefore, $f$ is an isomorphism of posets.

From the previous two propositions, we can directly deduce the lattice case of BPSZ, Theorem 2.2]: 
Corollary 2.5. Let $L$ be a lattice with finite length $l(L)<\infty$, and let $I \subset L$ be a proper ideal. Then $\Delta(\overline{\operatorname{Bier}(L, I)})$ is obtained from $\Delta(\bar{L})$ by a sequence of stellar subdivisions on all the edges from the set $\mathcal{S}=\{\{x, y\} \mid x \in I \backslash\{\hat{0}\}, y \in \bar{L} \backslash I\}$, where these subdivisions are performed in an order of increasing length $l(x, y)$.

Proof. If $\mathcal{G}$ is the building set defined in Proposition 2.1, then it is not hard to see that $\overline{\operatorname{Bier}(L, I)}=\mathcal{G} \cup \mathcal{S}^{\prime}$, where $\mathcal{S}^{\prime}=\{[x, y] \mid x \in I \backslash\{\hat{0}\}$ and $y \in \bar{L} \backslash I\}$. Let $\mathcal{S}=\left\{e_{1}, \ldots, e_{k}\right\}$, where if $e_{i}=\left\{x_{i}, y_{i}\right\}, e_{j}=\left\{x_{j}, y_{j}\right\}$, and $i<j \Rightarrow l\left(x_{i}, y_{i}\right) \leq$ $l\left(x_{j}, y_{j}\right)$. Set $\mathcal{G}_{0}=\mathcal{G}$, and for $i \in[k], \mathcal{G}_{i}=\mathcal{G}_{i-1} \cup\left\{\left[x_{i}, y_{i}\right]\right\}$, where $e_{i}=\left\{x_{i}, y_{i}\right\}$. It is clear that $\left[x_{i}, y_{i}\right] \in \max \left(\operatorname{Bier}(L, I)_{<\hat{1}} \backslash \mathcal{G}_{i-1}\right)$, and therefore, by Proposition 2.4 $\mathcal{F}\left(\mathcal{N}\left(\mathcal{G}_{i}\right)\right)=\mathrm{Bl}_{\left\{\left[x_{i}, \hat{1}\right],\left[\hat{o}, y_{i}\right]\right\}}\left(\mathcal{F}\left(\mathcal{N}\left(\mathcal{G}_{i-1}\right)\right)\right)$. In other words, since $\Delta(\bar{L})=\mathcal{N}\left(\mathcal{G}_{0}\right)$, and blowup in this case corresponds to stellar subdivision of the edge $\left\{x_{i}, y_{i}\right\}$, we have that

$$
\mathcal{N}\left(\mathcal{G}_{i}\right)=\operatorname{sd}_{e_{i}}\left(\operatorname{sd}_{e_{i-1}}\left(\ldots\left(\operatorname{sd}_{e_{1}} \Delta(\bar{L})\right)\right)\right) .
$$

We finish the proof remarking that $\mathcal{N}\left(\operatorname{Bier}(L, I)_{<\hat{1}}, \overline{\operatorname{Bier}(L, I)}\right)=\Delta(\overline{\operatorname{Bier}(L, I)})$, since $\overline{\operatorname{Bier}(L, I)}$ is the maximal building set in $\operatorname{Bier}(L, I)_{<\hat{1}}$.

It is important to emphasize the following:

Corollary 2.6. For any lattice $L$, and a proper ideal $I \subset L,\|\Delta(\overline{\operatorname{Bier}(L, I)})\|$ and $\|\Delta(\bar{L})\|$ are $P L$ homeomorphic. Furthermore, if $L$ is a face lattice of a strongly regular $P L C W$-sphere, then so is $\operatorname{Bier}(L, I)$.

\section{Recursive COATOM ORdeRINGS AND SHELlability of BieR lattices}

We now proceed to study the case of a shellable lattice or poset. After recalling the definition of shellability of a simplicial complex we will prove a proposition asserting that combinatorial blowups, and thus stellar subdivisions, preserve shellability.

Definition 3.1. Let $P$ be a bounded poset. We will say that $P$ admits a recursive coatom ordering if $P=\{\hat{0}, \hat{1}\}$, or if there exists a coatom ordering $c_{1}, \ldots, c_{r}$ such that the following two conditions are satisfied:

(R) For all $j \in[r]$, the poset $\left[\hat{0}, c_{j}\right]$ admits a recursive coatom ordering in which coatoms of $\left[\hat{0}, c_{j}\right]$ which are contained in $\left[\hat{0}, c_{i}\right]$, for some $i<j$, come before all other coatoms.

(S) For all $1 \leq i<k \leq r$ and all $x \in P$, if $x \leq c_{i}$ and $x \leq c_{k}$, then there exists some $j<k$ and some coatom $\omega$ of $\left[\hat{0}, c_{k}\right]$ so that $x \leq \omega \leq c_{j}$.

Remark. It was noticed in [Sha, Proposition 2.13] that, in the case when $L$ is a finite lattice and $c_{1}, \ldots, c_{r}$ is some coatom ordering of $L$, then this ordering satisfies condition (S) of Definition 3.1 if and only if it satisfies the following condition:

(T) For all $1 \leq i<j \leq r$, there exists some $k<j$ so that

$$
c_{i} \wedge c_{j} \leq c_{k} \wedge c_{j} \lessdot c_{j} .
$$

Since we will work with face lattices of simplicial complexes, we will verify conditions $(\mathrm{R})$ and $(\mathrm{T})$. The face lattice of a simplicial complex $K$ we will denote by $\hat{\mathcal{F}}(K)$, that is $\hat{\mathcal{F}}(K)=\mathcal{F}(K) \cup\{\hat{1}\}$.

We will now state two propositions which will be used further in the paper. For their proofs we refer the reader to the corresponding papers. 
Proposition 3.2 ([BW2, Theorem 5.13]). A simplicial complex $K$ is shellable if and only if $\hat{\mathcal{F}}(K)$ admits a recursive coatom ordering.

Proposition 3.3 ([BW1, Theorem 5.1]). A graded poset $P$ is totally semimodular if and only if for every interval $[x, y]$ of $P$, every atom ordering in $[x, y]$ is a recursive atom ordering.

The next theorem is the main result of this section.

Theorem 3.4. Let $\hat{\mathcal{F}}=\hat{\mathcal{F}}(K)$ be a face lattice of some simplicial complex $K$, $\mathcal{F}=\hat{\mathcal{F}} \backslash\{\hat{1}\}$, and assume that $\hat{\mathcal{F}}$ admits a recursive coatom ordering. Then $\hat{\mathcal{F}}_{\alpha}=$ $\mathrm{Bl}_{\alpha}(\mathcal{F}) \cup\{\hat{1}\}$ also admits a recursive coatom ordering, where $\alpha$ is any element of $\hat{\mathcal{F}} \backslash\{\hat{0}, \hat{1}\}$.

Proof. Since $\hat{\mathcal{F}}_{\alpha}$ is the face lattice of the simplicial complex $\operatorname{sd}_{\alpha}(K)$, intervals below maximal faces in $\hat{\mathcal{F}}_{\alpha}$ are boolean. Having in mind that every boolean lattice is selfdual, graded, and totally semimodular (and that a recursive atom ordering of a poset is a recursive coatom ordering of its dual), by Proposition 3.3 every coatom ordering of these intervals is recursive. Therefore, to check that some ordering of coatoms of $\hat{\mathcal{F}}_{\alpha}$ is recursive, it suffices to prove that it satisfies condition (T) from the remark above.

Suppose now that $\mathcal{O}=\left\{F_{1}, \ldots, F_{n}\right\}$ is a recursive coatom ordering of $\hat{\mathcal{F}}$. Define $\mathcal{C} \subset \mathcal{F}$ in the following way:

$$
\mathcal{C}=\max \{G \in \mathcal{F} \mid G \nsupseteq \alpha \text { and } G \cup \alpha \text { is an element of } \mathcal{F}\} .
$$

Set $I=\left\{i \in[n] \mid F_{i} \supseteq \alpha\right\}$. Then it is not hard to see that all coatoms of $\hat{\mathcal{F}}_{\alpha}$ are

$$
\operatorname{coat}\left(\hat{\mathcal{F}}_{\alpha}\right)=\left\{F_{i} \mid i \in[n] \backslash I\right\} \cup\{\langle\alpha, G\rangle \mid G \in \mathcal{C}\} .
$$

Denote the elements of $I$ by $i_{1}, \ldots, i_{t}$, where $t=|I|$, and $i_{1}<i_{2}<\cdots<i_{t}$. Having in mind that the $F_{i}$ 's are the coatoms of $\hat{\mathcal{F}}$, and that $F_{i} \supseteq \alpha$, for all $i \in I$, it is not hard to see that

$$
\mathcal{C}=\left\{G \in \mathcal{F} \mid G \nsupseteq \alpha \text { and } G \text { is a codimension } 1 \text { face of } F_{i} \text {, for some } i \in I\right\} .
$$

Now let, for all $l \in[n], \mathcal{A}_{l}=\left\{G \in \mathcal{C} \mid G \subseteq F_{l} \backslash\left(\bigcup_{j=1}^{l-1} F_{j}\right)\right\}$. It is easy to see that $\mathcal{C}=\bigsqcup_{1 \leq l \leq i_{t}} \mathcal{A}_{l}$, where the symbol $\bigsqcup$ denotes the disjoint union. For any $G \in \mathcal{C}$, let $A(G)=\min \left\{l \in[n] \mid F_{l} \supseteq G\right\}$ and let $\operatorname{mf}(G)=\min \left\{s \in I \mid G \lessdot F_{s}\right\}$. It is clear that $A(G) \leq \operatorname{mf}(G)$.

Define a relation $\prec$ between coatoms of $\hat{\mathcal{F}}_{\alpha}$ in the following way:

- For any $i, j \in[n] \backslash I, F_{i} \prec F_{j}$ if and only if $i<j$.

- For any $i \in[n] \backslash I$ and $G \in \mathcal{C}, F_{i} \prec\langle\alpha, G\rangle$ if and only if $i<\operatorname{mf}(G)$, and $F_{i} \succ\langle\alpha, G\rangle$ if and only if $i>\operatorname{mf}(G)$.

- For any $E, G \in \mathcal{C},\langle\alpha, E\rangle \prec\langle\alpha, G\rangle$ if and only if either $\operatorname{mf}(E)<\operatorname{mf}(G)$ or $\operatorname{mf}(E)=\operatorname{mf}(G)$ and $A(E)<A(G)$.

It is not hard to check that $\preceq$ is indeed a partial ordering. We will choose a linear extension thereof and denote it by $\mathcal{O}_{\alpha}$. In order to prove that condition (T) holds for $\mathcal{O}_{\alpha}$, we need to prove the following four cases:

(1) Assume $F_{i}, F_{j} \in \operatorname{coat}\left(\hat{\mathcal{F}}_{\alpha}\right)$, and let $F_{i}$ come before $F_{j}$ in $\mathcal{O}_{\alpha}$. Then, by definition, $i<j$, and $\alpha \nsubseteq F_{i}, F_{j}$. Since $\mathcal{O}$ is a recursive coatom ordering, there exists $k<j$ such that $F_{i} \cap F_{j} \subseteq F_{k} \cap F_{j}=F_{j} \backslash\{v\}$, where $v$ is some vertex of $F_{j}$. 
(1.1) If $\alpha \nsubseteq F_{k}$, then $F_{k} \in \operatorname{coat}\left(\hat{\mathcal{F}}_{\alpha}\right)$; clearly $F_{k}$ comes before $F_{j}$ in $\mathcal{O}_{\alpha}$, and $F_{i} \wedge F_{j} \leq F_{k} \wedge F_{j} \lessdot F_{j}$

(1.2) If $\alpha \subseteq F_{k}$, pick any vertex $w \in \alpha \backslash F_{j} \subset F_{k}$. Then $F_{k} \backslash\{w\}$ is a codimension 1 face of $F_{k}$ which does not contain $\alpha$, and therefore $\left\langle\alpha, F_{k} \backslash\{w\}\right\rangle \in \operatorname{coat}\left(\hat{\mathcal{F}}_{\alpha}\right)$. Noticing that $\operatorname{mf}\left(F_{k} \backslash\{w\}\right) \leq k<j$, it is clear that $\left\langle\alpha, F_{k} \backslash\{w\}\right\rangle$ comes before $F_{j}$ in $\mathcal{O}_{\alpha}$. Since $F_{j} \wedge\left\langle\alpha, F_{k} \backslash\{w\}\right\rangle=F_{j} \cap\left(F_{k} \backslash\{w\}\right)=F_{j} \backslash\{v\}$, we have that $F_{i} \wedge F_{j} \leq\left\langle\alpha, F_{k} \backslash\{w\}\right\rangle \wedge F_{j} \lessdot F_{j}$.

(2) Let $F_{i},\langle\alpha, G\rangle \in \operatorname{coat}\left(\hat{\mathcal{F}}_{\alpha}\right)$, and suppose that $F_{i}$ comes before $\langle\alpha, G\rangle$ in $\mathcal{O}_{\alpha}$. Then $\alpha \nsubseteq F_{i}$ and $i<\operatorname{mf}(G)$. Suppose first that $A(G)<\operatorname{mf}(G)$. Then $G \subseteq F_{A(G)}$, where $\alpha \nsubseteq F_{A(G)}$, and $F_{A(G)}$ comes before $\langle\alpha, G\rangle$ in $\mathcal{O}_{\alpha}$. Then we have that $F_{i} \wedge\langle\alpha, G\rangle=F_{i} \cap G \leq G=F_{A(G)} \cap G=F_{A(G)} \wedge\langle\alpha, G\rangle \lessdot\langle\alpha, G\rangle$, and condition $(\mathrm{T})$ is satisfied in this case.

Now let $A(G)=\operatorname{mf}(G)=j$. By the assumption, there exists $k<j$ such that $F_{i} \cap F_{j} \subseteq F_{k} \cap F_{j}=F_{j} \backslash\{v\}$, for some $v \in F_{j}$. Note that $v \in G$, since otherwise $G \subseteq F_{k}$, and therefore $A(G) \leq k<j$, which would give a contradiction.

Since $G \lessdot F_{j}$, denote by $w$ the vertex such that $F_{j} \backslash\{w\}=G$, where clearly $w \in \alpha$, and let

$$
H= \begin{cases}F_{j} \backslash\{v\} \lessdot F_{j}, & \text { if } \alpha \nsubseteq F_{k} ; \\ F_{k} \backslash\{w\} \lessdot F_{k}, & \text { if } \alpha \subseteq F_{k} .\end{cases}
$$

By simple checking, one can see that $\alpha \nsubseteq H$, and $H \in \mathcal{C}$, since $H$ is a coatom in some face that contains $\alpha$. Seeing that $H \subseteq F_{k}$, we conclude that either $\operatorname{mf}(H)<$ $\operatorname{mf}(G)$, or $\operatorname{mf}(H)=\operatorname{mf}(G)$ and $A(H)<A(G)$, and therefore $\langle\alpha, H\rangle$ comes before $\langle\alpha, G\rangle$ in $\mathcal{O}_{\alpha}$. Now it is easy to see that

$$
F_{i} \wedge\langle\alpha, G\rangle=F_{i} \cap G \leq G \backslash\{v\}<\langle\alpha, G \backslash\{v\}\rangle=\langle\alpha, G\rangle \wedge\langle\alpha, H\rangle \lessdot\langle\alpha, G\rangle .
$$

(3) If $\langle\alpha, G\rangle, F_{j} \in \operatorname{coat}\left(\hat{\mathcal{F}}_{\alpha}\right)$ and $\langle\alpha, G\rangle$ comes before $F_{j}$ in $\mathcal{O}_{\alpha}$, then it must be $\operatorname{mf}(G)<j$. The proof for this case is the same as for case (1), having in mind that $\langle\alpha, G\rangle \wedge F_{j}=G \cap F_{j} \leq F_{\operatorname{mf}(G)} \cap F_{j}$, and since in case (1) we didn't use the fact that $\alpha \nsubseteq F_{i}$.

(4) Finally, the case when $\langle\alpha, E\rangle,\langle\alpha, G\rangle \in \operatorname{coat}\left(\hat{\mathcal{F}}_{\alpha}\right)$, and $\langle\alpha, E\rangle$ comes before $\langle\alpha, G\rangle$ in $\mathcal{O}_{\alpha}$. Then there are two possibilities, either $\operatorname{mf}(E)=\operatorname{mf}(G)$ or $\operatorname{mf}(E)<$ $\operatorname{mf}(G)$.

The case when $j:=\operatorname{mf}(E)=\operatorname{mf}(G)$ is easy, namely both $E$ and $G$ are codimension 1 faces in $F_{j}$, and hence $E \cap G$ is a codimension 1 face of $G$. Therefore, we have that $\langle\alpha, E\rangle \wedge\langle\alpha, G\rangle=\langle\alpha, E \cap G\rangle \lessdot\langle\alpha, G\rangle$.

If $\operatorname{mf}(E)<\operatorname{mf}(G)$, let $i=\operatorname{mf}(E), j=\operatorname{mf}(G)$, and let $k<j$ be an integer such that $F_{i} \cap F_{j} \subseteq F_{k} \cap F_{j}=F_{j} \backslash\{v\}$, for some $v \in F_{j}$. Define $w$ to be the vertex such that $F_{j} \backslash\{w\}=G$, and $H$ as in equation (3.1). Then, since $E \cap G \subseteq F_{i} \cap G \subseteq G \backslash\{v\}$, we have that

$$
\langle\alpha, E\rangle \wedge\langle\alpha, G\rangle=\langle\alpha, E \cap G\rangle \leq\langle\alpha, G \backslash\{v\}\rangle=\langle\alpha, G\rangle \wedge\langle\alpha, H\rangle \lessdot\langle\alpha, G\rangle .
$$

Therefore we have proved that $\mathcal{O}_{\alpha}$ satisfies condition $(\mathrm{T})$ and hence is a recursive coatom ordering of $\hat{\mathcal{F}}_{\alpha}$.

Since stellar subdivisions are described by combinatorial blowups in the face poset, we can formulate the following corollary.

Corollary 3.5. If $K$ is a shellable simplicial complex, then so is $\operatorname{sd}_{F}(K)$, where $F$ is any non-empty face of $K$. 
Now we return to the general Bier poset construction, and conclude that:

Corollary 3.6. Let $L$ be a lattice so that $\Delta(\bar{L})$ is shellable. If $I \subset L$ is a proper ideal in $L$, then $\Delta(\overline{\operatorname{Bier}(L, I)})$ is also shellable.

Remark 3.7 (The general case). If we consider any bounded poset $P$, the first part of Corollary 2.6 remains true (see [BPSZ, Corollaries 2.3 and 2.4] for a proof of this fact). Since stellar subdivisions are described by combinatorial blowups in the face poset, Corollary 3.6 also remains true in the general case.

Let us also mention here that, by a remark at the end of section 2 and BPSZ, Theorem 2.2], for every bounded poset $P$ and for any proper ideal $I \subset P$, the simplicial complexes $\Delta(\bar{P})$ and $\Delta(\overline{\operatorname{Bier}(P, I)})$ have the same simple homotopy type.

Remark 3.8 (Shellable spheres). It is clear that by using the Bier poset construction together with Corollaries 2.6 and 3.6. one can obtain numerous simplicial shellable $n$-spheres with more than $2(n+2)$ vertices, therefore answering one of the questions asked in BPSZ.

Choosing an appropriate poset $L$ we also obtain numerous shellable simplicial complexes with the homotopy type of wedges of spheres, with any number of vertices.

\section{ACKNOWLEDGMENTS}

We would like to thank Eva Maria Feichtner and Dmitry Kozlov for pointing out this problem to us, and for their useful suggestions and comments. We also thank the anonymous referee for his suggestions that improved the presentation of the paper.

\section{REFERENCES}

[B] T. Bier, A remark on Alexander duality and the disjunct join, Preprint (1992).

[BPSZ] A. Björner, A. Paffenholz, J. Sjöstrand, G.M. Ziegler, Bier spheres and posets, Discrete Comput. Geom. 34 (2005), no. 1, 71-86 MR.2140883 (2006k:06005)

[BW1] A. Björner, M. Wachs, On lexicographically shellable posets, Trans. Amer. Math. Soc. 277 (1983), no. 1, 323-341. MR0690055 (84f:06004)

[BW2] A. Björner, M. Wachs, Shellable nonpure complexes and posets. I, Trans. Amer. Math. Soc. 348 (1996), no. 4, 1299-1327. MR1333388 (96i:06008)

[DCP] C. De Concini, C. Procesi, Wonderful models of subspace arrangements, Selecta Math. (N.S.) 1 (1995), no. 3, 459-494. MR.1366622 (97k:14013)

[F1] E.M. Feichtner, De Concini-Procesi arrangement models - a discrete geometer's point of view in: Combinatorial and Computational Geometry, J.E. Goodman, J. Pach, E. Welzl, eds; MSRI Publications 52, Cambridge University Press, 2005, 333-360.

[F2] E.M. Feichtner, Complexes of trees and nested set complexes, to appear in Pacific J. Math. arXiv: math.CO/0409235 v2

[FM] E.M. Feichtner, I. Müller, On the topology of nested set complexes, Proc. Amer. Math. Soc. 133 (2005), no. 4, 999-1006 (electronic). MR 2117200 (2006c:06005)

[FK1] E.M. Feichtner, D.N. Kozlov, Incidence combinatorics of resolutions, Selecta Math. (N.S.) 10 (2004), no. 1, 37-60. MR2061222

[FK2] E.M. Feichtner, D.N. Kozlov, A desingularization of real differentiable actions of finite groups, Int. Math. Res. Not. 2005, no. 15 (2005), 881-898. MR2147091 (2006e:14063)

[FS] E.M. Feichtner, B. Sturmfels, Matroid polytopes, nested sets and Bergman fans, to appear in Port. Math. (N.S.), arXiv:math.Co/0411260 MR2191630 (2006j:05036)

[Ka] G. Kalai, Many triangulated spheres, Discrete Comput. Geom. 3 (1988), no. 1, 1-14. MR 0918176 (89b:52025)

[Ko1] D.N. Kozlov, General lexicographic shellability and orbit arrangements, Ann. Comb. 1 (1997), no. 1, 67-90. MR.1474801 (98h:52023) 
[Ko2] D.N. Kozlov, Simple homotopy types of Hom-complexes, neighborhood complexes, Lovász complexes, and atom crosscut complexes, to appear in Topology and its Applications, arXiv: math.AT/0503613

[M] J. Matoušek, Using the Borsuk-Ulam theorem, Springer Universitext, Springer Verlag, Berlin, 2003. MR1988723 (2004i:55001)

[Sha] J. Shareshian, On the shellability of the order complex of the subgroup lattice of a finite group, Trans. Amer. Math. Soc. 353 (2001), no. 7, 2689-2703 (electronic). MR1828468 (2002k:06006)

[Sta] R. Stanley, Enumerative combinatorics, Vol. 1, Wadsworth and Brooks/Cole, Monterey, CA, 1986; reprinted as Cambridge Studies in Advanced Mathematics, Vol. 49, Cambridge University Press, Cambridge, 1997. MR.1442260 (98a:05001)

Institute of Theoretical Computer Science, ETH Zurich, 8092 Zurich, Switzerland

E-mail address: sonja@math.binghamton.edu

Department of Mathematics, ETH Zurich, 8092 Zurich, Switzerland

E-mail address: delucchi@mail.dm.unipi.it 